Article

\title{
An Inverse Synthetic Aperture Ladar Imaging Algorithm of Maneuvering Target Based on Integral Cubic Phase Function-Fractional Fourier Transform
}

\author{
Yakun Lv ${ }^{1, *(D)}$, Yanhong Wu ${ }^{1}$, Hongyan Wang ${ }^{2}$, Lei Qiu ${ }^{1}$, Jiawei Jiang ${ }^{1}$ and Yang Sun ${ }^{3}$ (D) \\ 1 Department of Electronic and Optical Engineering, Space Engineering University, Beijing 101416, China; \\ mail2wyh@163.com (Y.W.); qiulei2013@nudt.edu.cn (L.Q.); herojjw@163.com (J.J.) \\ 2 School of Space Information, Space Engineering University, Beijing 101416, China; yhgnaw@163.com \\ 3 Science and Technology on Complex Electronic System Simulation Laboratory, Beijing 101416, China; \\ fireflypd@buaa.edu.cn \\ * Correspondence: qiul06@mails.tsinghua.edu.cn; Tel.: +86-010-6636-5117
}

Received: 11 July 2018; Accepted: 13 August 2018; Published: 15 August 2018

\begin{abstract}
When imaging maneuvering targets with inverse synthetic aperture ladar (ISAL), dispersion and Doppler frequency time-variation exist in the range and cross-range echo signal, respectively. To solve this problem, an ISAL imaging algorithm based on integral cubic phase function-fractional Fourier transform (ICPF-FRFT) is proposed in this paper. The accurate ISAL echo signal model is established for a space maneuvering target that quickly approximates the uniform acceleration motion. On this basis, the chirp rate of the echo signal is quickly estimated by using the ICPF algorithm, which uses the non-uniform fast Fourier transform (NUFFT) method for fast calculations. At the best rotation angle, the range compression is realized by FRFT and the range dispersion is eliminated. After motion compensation, separation imaging of strong and weak scattering points is realized by using ICPF-FRFT and CLEAN technique and the azimuth defocusing problem is solved. The effectiveness of the proposed method is verified by a simulation experiment of an aircraft scattering point model and real data.
\end{abstract}

Keywords: inverse synthetic aperture ladar (ISAL); maneuvering target; integral cubic phase function (ICPF); fractional Fourier transform (FRFT); non-uniform fast Fourier transform (NUFFT); CLEAN technique

\section{Introduction}

With the development of radar detection technology, high-precision target imaging has become an important aspect of the detection task. Inverse synthetic aperture ladar (ISAL) combines coherent laser technology and inverse synthetic aperture technology, overcoming the limitations of the actual aperture and diffraction. ISAL also overcomes the shortfalls of traditional microwave imaging radars that cannot provide enough range resolution for remote target and small target imaging and solves the problem experienced by traditional laser imaging radar, which cannot perform the high-resolution imaging of a moving target [1]. ISAL is the only optical means by which centimeter-level resolution can be obtained at a range of thousands of kilometers [2]. Therefore, ISAL imaging can fulfil the requirement for high precision imaging and quasi real-time imaging for target surveillance.

ISAL imaging is similar to the traditional inverse synthetic aperture radar (ISAR) imaging principle but due to the use of laser as a radiation source, ISAL has an ultra-high carrier frequency, ultra-large bandwidth and extremely short wavelength. Compared with ISAR, ISAL has higher resolution, smaller imaging angle and shorter imaging time [3]. Research on ISAL has mainly focused on principal analysis and algorithm simulation. Some close-range field tests have been reported [4-8]. 
Research on space target imaging using the space-based platform ISAL is still in its infancy, so the research in this area is important.

ISAL uses an ultra-high carrier frequency and ultra-large bandwidth signal and the existing range compression method disperses and distorts the echo range profile. In addition, the ISAL azimuth Doppler of the maneuvering target is time variant. If the conventional azimuth image method is used, the imaging quality will be seriously defocused. Two kinds of methods are currently available for imaging the maneuvering target in the microwave section ISAR: parameter estimation and time-frequency analysis. The parameter estimation method models the signal echo into a multi-component signal model, then uses the signal estimation method to estimate the maneuvering parameters of the signal and then compensates the signal after the estimation. The commonly used estimation methods include maximum likelihood (ML) estimation [9], high-order ambiguity function (HAF) [10], discrete chirp Fourier transform (DCFT) [11] and a variety of improved algorithms [12]. The disadvantage with these methods is that it is generally required to search for parameters and the computational complexity is considerable for high precision estimation. The time-frequency analysis method involves the instantaneous Doppler, which obtains the signal using the time frequency analysis method and then uses range instantaneous Doppler (RID) for imaging. The common RID methods include short time Fourier transform (STFT) [13], wavelet transform, S transform, Wigner-Ville distribution (WVD), the smoothed pseudo WVD (SPWVD) [14] and the adaptive time-frequency decomposition method $[15,16]$. The disadvantage of these methods is that the time-frequency resolution is low and cross terms exist, which affects the imaging quality. Given the high-resolution capability of ISAL, a large amount of data is generated. Therefore, finding a fast and efficient imaging method suitable for ISAL imaging is necessary.

In this study, to solve the above problems, we first analyzed the exact echo model of the target. Secondly, given the problem of the one-dimensional (1D) range dispersion of the echo signal, the frequency modulation rate of the echo signal was quickly estimated using the ICPF algorithm and then used the modulation frequency to calculate the best rotation angle in the FRFT domain. At this angle, the FRFT [17] method was used to achieve the range compression and eliminate the range dispersion. Then, to address the problem of azimuth defocus, ICPF combined with the CLEAN technique was proposed to estimate the frequency modulation rate of the strong and weak scattering points and then the azimuth compression imaging was realized using FRFT. Finally, the effectiveness of the method was verified by a simulation experiment of the plane scattering point model.

\section{ISAL Signal Echo Model}

The three-dimensional (3D) imaging geometry of the maneuvering target is shown in Figure 1a. Where the coordinate origin $\mathrm{O}$ is the target turntable center, point $P\left(x_{p}, y_{p}, z_{p}\right)$ is any scattering point on the target and $r_{p}$ is the scattering point $P$ position vector starting from $\mathrm{O}$. $\omega$ is the rotational angular velocity vector of the target three-dimensional motion. The ISAL imaging projection plane $\Gamma$ is determined by the vector $\omega$ and the radar line-of-sight direction (LOS) unit vector $\mathbf{R}, \omega$ can be decomposed into the radial rotational component, $\omega_{r}$ along the LOS and the rotational component, $\omega_{e}$ perpendicular to the LOS. $\omega_{r}$ cannot cause the radial movement of the scattering point, that is, it will not cause the phase change of the echo and $\omega_{e}$ will cause the scattering point to move radially, resulting in Doppler frequency variation, which can achieve high-resolution ISAL imaging of the target, $\omega_{e}$ is called effective rotation component. The three-dimensional motion velocity of the target can be decomposed into a component $v$ in the $\Gamma$ plane and a component perpendicular to the $\Gamma$ plane and the vertical component does not affect the imaging of the target, so this component can be ignored. For the parallel component $v$, it can be decomposed into the radial component $v_{r}$ along the LOS and the component $v_{e}$ perpendicular to the LOS. $v_{r}$ causes the Doppler shift of the target echo which cause phase change, while $v_{e}$ does not generate Doppler shift.

After the above analysis, the effective component in the three-dimensional (3D) imaging geometry can be projected onto the imaging plane $\Gamma$ to obtain a two-dimensional (2D) turntable imaging geometry 
as shown in Figure $1 \mathrm{~b}$ [18]. In two-dimensional (2D) imaging geometry, only the relative motion between radar and target is considered. Where $\mathrm{O}$ is the reference point and $\mathrm{XOY}$ is the rectangular coordinate system fixed on the target, the $\mathrm{Y}$ axis is the direction of the radar LOS. The target moves along the $Y$ axis with the speed of $v_{r}$ and rotates around the $\mathrm{O}$ point at the angular velocity of $\omega_{e}$. Suppose that at time $t\left(t\right.$ is full-time and satisfies the equation $t=t_{k}+t_{m}$, where $t_{k}$ is the range fast time and $t_{m}$ is the azimuth slow time, $\left.m=1,2, \ldots, M\right)$, the range between the target geometry center and the radar is $R_{0}(t)$. The rotation angle of the target relative to the radar is $\theta(t)$. Then, the range $R_{P}(t)$ between any point $P(x, y)$ in the target and the radar is:

$$
R_{P}(t)=\sqrt{R_{0}(t)^{2}+r_{p}^{2}-2 R_{0} r_{p} \cos \left[\theta(t)+\frac{\pi}{2}\right]} \approx R_{0}(t)+x \sin \theta(t)+y \cos \theta(t)
$$

Considering the inertia of the conventional target motion and the short imaging time of ISAL, which is less than $1 \mathrm{~s}$, maneuvering target motion can only approximate to the second-order motion component. In other words, $R_{0}(t)$ and $\theta(t)$ can be approximated as the second-order function of $t^{2}$ :

$$
\begin{gathered}
R_{0}(t)=R_{0}+v_{0} t+\frac{1}{2 !} a t^{2} \\
\theta(t)=\theta_{0}+\omega t+\frac{1}{2 !} \Omega t^{2}
\end{gathered}
$$

where $R_{0}$ is the initial range, $v_{0}$ is the initial radial velocity, $a$ is the radial acceleration, $\theta_{0}$ is the initial rotation angle, $\omega$ is the rotation angular velocity and $\Omega$ is the rotation angular acceleration.

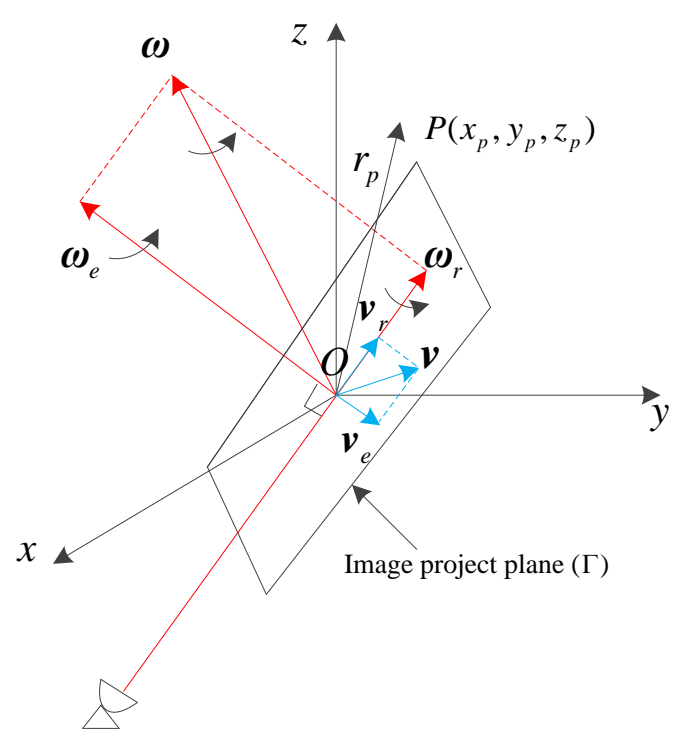

(a)

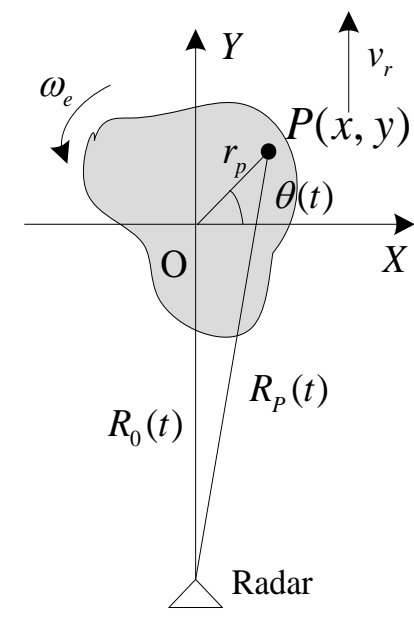

(b)

Figure 1. Inverse synthetic aperture ladar (ISAL) turntable imaging geometry; (a) three-dimensional (3D) imaging geometry; (b) two-dimensional (2D) imaging geometry.

Because wavelength of ISAL is on the micron scale, it is sensitive to the motion of the target, so the effect of the fast time $t_{k}$ on the radial range cannot be ignored [19]. When the pulse duration is short, the influence of $t_{k}$ on rotation components can be neglected. So, Equations (2) and (3) can be resolved as:

$$
\begin{aligned}
R_{0}(t) & =R_{0}+v_{0}\left(t_{m}+t_{k}\right)+\frac{1}{2} a\left(t_{m}+t_{k}\right)^{2} \\
& =R_{0}+v_{0} t_{m}+\frac{1}{2} a t_{m}^{2}+\left(v_{0}+a t_{m}\right) t_{k}+\frac{1}{2} a t_{k}^{2} \\
& =R_{0}\left(t_{m}\right)+v\left(t_{m}\right) t_{k}+\frac{1}{2} a t_{k}^{2}
\end{aligned}
$$




$$
\theta(t) \approx \theta\left(t_{m}\right)=\theta_{0}+\omega t_{m}+\frac{1}{2} \Omega t_{m}^{2}
$$

where $R_{0}\left(t_{m}\right)$ and $v\left(t_{m}\right)$ represent the radial range and velocity varies with the azimuth time $t_{m}$, respectively.

According to the above equation, Equation (1) can be rewritten as:

$$
R_{P}(t) \approx R_{0}\left(t_{m}\right)+R_{d}\left(t_{m}\right)+v\left(t_{m}\right) t_{k}+\frac{1}{2} a t_{k}^{2}
$$

where $R_{d}\left(t_{m}\right)=x \sin \theta\left(t_{m}\right)+y \cos \theta\left(t_{m}\right)$.

ISAL usually uses ultra-bandwidth Linear Frequency Modulated (LFM) signals to achieve high range resolution, the expression of the transmitted signal can be written as:

$$
\begin{gathered}
s_{t}\left(t_{k}, t_{m}\right)=\operatorname{rect}\left(\frac{t_{k}}{T_{p}}\right) \exp \left(j 2 \pi f_{c} t+j \pi k t_{k}^{2}\right) \\
\operatorname{rect}\left(\frac{t_{k}}{T_{p}}\right)=\left\{\begin{array}{c}
1,\left|t_{k}\right| \leq T_{p} / 2 \\
0,\left|t_{k}\right|>T_{p} / 2
\end{array}\right.
\end{gathered}
$$

where $T_{p}$ is the width of the pulse, $f_{c}$ is the carrier frequency and $k$ is the chirp-rate.

Considering $c \gg v_{r}+a t$, we ignore the effect of the target speed of the irradiating and receiving radar signals. Suppose the radar receives the echo signal of point $P$ after time delay $\tau=2 R_{p}(t) / c$, the radar receiving signal is:

$$
s_{r}\left(t_{k}, t_{m}\right)=\operatorname{\sigma rect}\left(\frac{t_{k}-\tau}{T_{p}}\right) \exp \left(j 2 \pi f_{c}\left(t_{k}-\tau\right)+j \pi k\left(t_{k}-\tau\right)^{2}\right)
$$

To reduce the data rate, ISAL often uses optical heterodyne coherent detection [20] to handle the echo signals. Suppose the reference delay of the coherent pulses is $\tau_{r e f}=2 R_{r e f}\left(t_{m}\right) / c$, the reference signal is:

$$
s_{r e f}\left(t_{k}, t_{m}\right)=\operatorname{rect}\left(\frac{t_{k}-\tau_{r e f}}{T_{p}}\right) \exp \left(j 2 \pi f_{c}\left(t-\tau_{r e f}\right)+j \pi k\left(t_{k}-\tau_{r e f}\right)^{2}\right)
$$

Therefore, the output signal after optical heterodyne detection is:

$$
\begin{aligned}
& s_{\mathrm{O}}\left(t_{k}, t_{m}\right)=s_{\mathrm{r}}\left(t_{k}, t_{m}\right) \cdot s^{*}{ }_{\text {ref }}\left(t_{k}, t_{m}\right) \\
& =\sigma_{p} r e c t\left(\frac{t_{k}-\tau}{T_{p}}\right) \exp \left[j 2 \pi f_{c}\left(\tau_{r e f}-\tau\right)\right] \cdot \exp \left[-j \pi k\left(\tau_{r e f}^{2}-\tau^{2}\right)\right] \cdot \exp \left[j 2 \pi k\left(\tau_{r e f}-\tau\right) t_{k}\right]
\end{aligned}
$$

We substitute Equations (4) and (6) into Equation (11) to obtain a polynomial function about time $t_{k}$ :

$$
\begin{gathered}
s_{o}\left(t_{k}, t_{m}\right)=\sigma_{p} \operatorname{rect}\left(\frac{t_{k}-\tau}{T_{p}}\right) \cdot \exp \left[j 2 \pi\left(P_{0}+P_{1} t_{k}+P_{2} t_{k}^{2}+P_{3} t_{k}^{3}+P_{4} t_{k}^{4}\right)\right] \\
P_{0}=2 k \frac{\Delta R_{m P}\left(\Delta R_{m P}+2 R_{r e f}\left(t_{m}\right)\right)}{c^{2}}-2 f_{c} \frac{\Delta R_{m P}}{c} \\
P_{1}=v\left(t_{m}\right)\left(4 k \frac{R_{0}\left(t_{m}\right)+R_{d}\left(t_{m}\right)}{c^{2}}-\frac{2 f_{c}}{c}\right)-\frac{2 k}{c} \Delta R_{m P} \\
P_{2}=-\frac{a f_{c}}{c}+2 a k \frac{R_{0}\left(t_{m}\right)+R_{d}\left(t_{m}\right)}{c^{2}}+2 k \frac{v\left(t_{m}\right)^{2}}{c^{2}}-2 k \frac{v\left(t_{m}\right)}{c} \\
P_{3}=2 a k_{r} \frac{v\left(t_{m}\right)}{c^{2}}-\frac{a k_{r}}{c} \\
P_{4}=\frac{k_{r} a^{2}}{2 c^{2}}
\end{gathered}
$$


where $\Delta R_{m P}=R_{0}\left(t_{m}\right)+R_{d}\left(t_{m}\right)-R_{r e f}\left(t_{m}\right)$. When $R_{r e f}\left(t_{m}\right)$ is accurately estimated, it is approximately equal to $R_{0}\left(t_{m}\right)$, then $\Delta R_{m P}=R_{d}\left(t_{m}\right)$, which is only related to the scattering point on the target in azimuth time. In practice, considering the impact of the target velocity on $\tau$ and $\tau_{\text {ref }}$, the envelope in the Equation (12) will cause a contraction in time. But the impact does not affect the analysis of the range dispersion, which is ignored here [1]. $P_{0}$ is the phase term related only to the azimuth time $t_{m}$, in which $2 f_{c} \Delta R_{m P} / c$ is a necessary term for azimuth compression and has no influence on range compression. $P_{1}$ is the first-order phase term. The first item in $P_{1}$ is mainly affected by the high carrier signal frequency, which produces the signal in the pulse Doppler. For the uniformly accelerated moving target, the Doppler coupling time shift with azimuth time change is produced, resulting in a range move. The second item contains the range information $2 k \Delta R_{m P} / c$, which is key to attaining range compression. $P_{2}$ is the chirp-rate phase term, mainly influenced by the ultra-high carrier frequency and large bandwidth. It is the root cause of the division and broadening of the peaks of the range. The range dispersion effect occurs if the conventional DFT is used for the compression processing of the range direction. From the expression $P_{2}$, the chirp-rate term of all scattering points in the single pulse echo is the same fixed value, whereas for different pulse echoes, the chirp-rate rate varies with slow time $t_{m}$. Therefore, processing the pulse echo sequence one at a time is necessary. $P_{3}$ and $P_{4}$ are the high-order phase terms. Because in a pulse period $c \gg a v\left(t_{m}\right), T_{p}{ }^{2} \ll c / 2 k_{r} T_{p}$ and $T_{p}{ }^{3} a^{2} \ll c^{2} / 2 k_{r} T_{p}$, the influence of the $P_{3}$ and $P_{4}$ on the intra-pulse Doppler spectrum broadening can be ignored.

According to the above analysis, in the imaging time, the second-order polynomial approximation can appropriately reflect the motion state of the maneuvering target and meet the imaging needs. The effect of the third- and fourth-order terms can be ignored. The range echo signals after heterodyne detection can be approximated to multicomponent LFM signals with the same frequency modulation slope:

$$
s_{o}\left(t_{k}, t_{m}\right)=\sigma_{p} \operatorname{rect}\left(\frac{t_{k}-\tau}{T_{p}}\right) \cdot \exp \left[j 2 \pi\left(P_{0}+P_{1} t_{k}+P_{2} t_{k}^{2}\right)\right]
$$

\section{Range Imaging Based on ICPF-FRFT}

FRFT is a kind of generalized Fourier transform that better focuses LFM signals [21]. The FRFT of the signal $s(t)$ is defined as:

$$
S_{\alpha}(u)=F^{p}[s(t)]=\int_{-\infty}^{\infty} s(t) K_{\alpha}(t, u) d t
$$

where $p$ is the order of the FRFT, which can be any real number and $\alpha$ is the rotation angle; $\alpha=p \pi / 2$. When $\alpha=\pi / 2$, FRFT becomes a traditional Fourier transform. $K_{\alpha}(t, u)$ is the transformation operator, the expression is:

$$
K_{\alpha}(t, u)=\left\{\begin{array}{l}
\sqrt{1-j \cot \alpha} \exp \left(j \pi\left(\left(t^{2}+u^{2}\right) \cot \alpha-2 u t \csc \alpha\right)\right) \\
\alpha \neq n \pi ; \\
\delta(t-u), \quad \alpha=2 n \pi \\
\delta(t+u), \quad \alpha=(2 n \pm 1) \pi \\
\alpha \neq n \pi ;
\end{array}\right.
$$

FRFT is equivalent to projecting the signal on the frequency axis after the counterclockwise rotation $\alpha$ of the signal in the time-frequency plane. When the $u$ axis of the FRFT is rotated to the time-frequency ridge of the signal, the amplitude of the signal projection to the fractional frequency $u$ axis is maximized and the rotation angle at this time is called the best angle $\alpha_{k}$ of rotation. Therefore, the projection of FRFT at the best angle of rotation can be used for range imaging and the imaging principle is shown in Figure 2. 


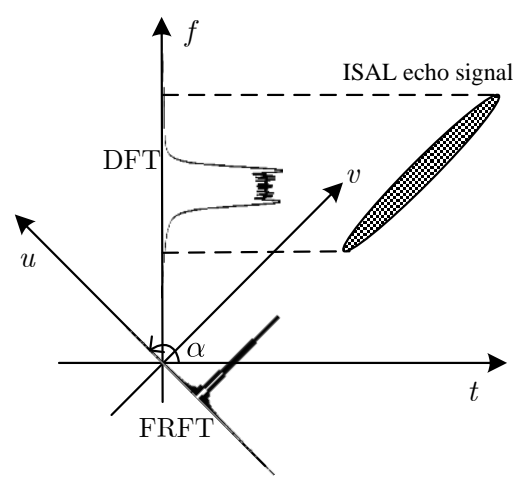

Figure 2. Schematic diagram of range compression via Discrete Fourier transform (DFT) and fractional Fourier transform (FRFT).

FRFT requires the peak search method in the two-dimensional (2D) plane $(\alpha, u)$ to obtain the optimal rotation angle. Therefore, the effect of range image compression depends on the value of $\alpha_{k}$ and its precision is easily influenced by the resolution of the search angle. The computation requirement is considerable in the high precision search. So, accuracy and computation are difficult to achieve. Paper and colleagues [22-25] proposed that ICPF can quickly estimate the chirp rate of LFM signals. The method only requires a 1D search and has good anti-noise performance and high estimation accuracy without being affected by subjective factors such as search resolution. Therefore, in this paper, ICPF was firstly used to estimate the modulation frequency of the optical heterodyne output signal and then the optimal rotation angle and the order of the FRFT were calculated. Finally, the range compression imaging was completed by FRFT at the optimal rotation angle. The ICPF definition of signal $x(t)$ is as follows:

$$
\operatorname{ICPF}(\mu)=\frac{1}{T} \int_{-\infty}^{\infty}\left|\int_{-\infty}^{\infty} x(t+\tau) x(t-\tau) \exp \left(-j \mu \tau^{2}\right) d \tau\right|^{2} d t
$$

From the definition, ICPF is a kind of transformation that detects the chirp-rate of the signal, which can concentrate the signal energy on the chirp-rate of the signal, in line with the energy distribution of the linear frequency modulation signal. Since ICPF needs to calculate the $\tau^{2}$ of the signal, using FFT for fast calculations is not possible. Therefore, we used the non-uniform fast Fourier transform (NUFFT) [26,27] to overcome the rigorous data sampling requirements of the FFT and to improve the algorithm's calculation speed. The NUFFT is defined as:

$$
\hat{z}_{k}=\sum_{l=1}^{M} z_{l} \exp \left(-j 2 \pi x_{l} / N\right), k=-N / 2, \cdots, N / 2+1
$$

where $z_{l}$ is non-uniform sampling time and $x_{l}$ is the corresponding non-uniform sampling position. Here, interpolation time domain non-uniform sampling data $z_{l}$ is replaced by an interpolation index term to achieve fast non-uniform Fourier transform.

$$
\text { Suppose that } \hat{\varphi}(x)=\sqrt{\frac{2}{\pi}} \frac{\sinh \left(\alpha \sqrt{K^{2}-x^{2}}\right)}{\sqrt{K^{2}-x^{2}}},-K \leq x \leq K, \hat{\varphi}(\xi)=\left\{\begin{array}{c}
I_{0} K \sqrt{\alpha^{2}-\xi^{2}},|\xi| \leq \alpha \\
0, \quad|\xi|>\alpha
\end{array},\right. \text { where, }
$$

$K$ is the length of interpolation kernel function. According to P. O'Shea [27], the exponential function can be expanded as shown in Equation (23):

$$
\exp (-j x \xi)=\frac{1}{\sqrt{2 \pi} \varphi(\xi)} \sum_{m \in Z} \hat{\varphi}(x-m) \exp (-j m \xi)
$$


where $|\xi| \leq \pi / c, c$ is the oversampling factor. Suppose $x=c x_{1}, \xi=2 \pi k /(c N),|k| \leq N / 2$, $\alpha=\pi(2-1 / c)-0.01, \varphi_{k}=\varphi(2 \pi k /(c N))$ and $\hat{\varphi}_{l m}=\frac{1}{2 \pi} \hat{\varphi}\left(c x_{l}-\left(\mu_{l}+m\right)\right)$. Substituting Equation (23) into Equation (22) yields a uniform frequency output:

$$
\hat{z}_{k}=\frac{1}{\varphi_{k}} \sum_{j=-c N / 2}^{c N / 2-1} u_{j} \exp (-j 2 \pi k j /(c N))
$$

where

$$
u_{j}=\sum_{l=1}^{M} \sum_{m \in Z} z_{l} \hat{\varphi}_{l, j+c m N-\mu_{l}, j}, j=-c N / 2, \cdots, c N / 2-1
$$

The specific NUFFT implementation process is shown in Figure 3. First, the intermediate parameters $\mu_{l}, \varphi_{k}, \hat{\varphi}_{l m}$ are calculated from the input non-uniform sampling data $z_{l}$ and the corresponding position $x_{l}$. Then, the intermediate variable $u_{j}$ is calculated according to Equations (25). Finally, the corresponding frequency output value $\hat{z}_{k}$ is calculated using Equation (24) with fast FFT.

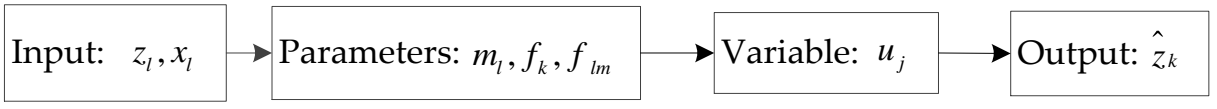

Figure 3. Non-uniform fast Fourier transform (NUFFT) diagram.

According to the above NUFFT principle, the non-uniform Fourier transform in the proposed ICPF is quickly implemented with NUFFT to reduce the computational complexity of the algorithm, so Equation (26) can be written as:

$$
\operatorname{ICPF}(\mu)=\operatorname{FFT}_{t}\left[\operatorname{NUFFT} T_{\tau^{2}}\left(\operatorname{ICPF}\left(t, \mu_{\tau^{2}}\right)\right)\right]
$$

where NUFFT $\tau_{\tau^{2}}$ indicates the NUFFT operation on the variable $\tau^{2}$ and $F F T_{t}$ indicates FFT operation on the variable $t$.

Assuming $N$ is the sampling point of a single pulse echo and $M$ is the number of DFT search points, the complexity of the ICPF-based DFT direct calculation is $\mathrm{O}\left(\mathrm{N}^{2} \mathrm{M}\right)$, whereas the complexity of the non-uniform Fourier transform calculation method is $\mathrm{O}\left(\mathrm{Nlog}_{2}(\mathrm{~N})\right)$ [28]. Assuming that $K$ is the number of scanning points in the FRFT transform domain $\alpha$, which is determined by the step size and range of $\alpha$, the complexity of the discrete FRFT is $\mathrm{O}\left(\mathrm{NKlog}_{2}(\mathrm{~N})\right)$. If we want an accurate $\alpha_{k}, \mathrm{~K}$ is usually large. The transformation and 2D search need to be coordinated [21,24], so the proposed NUFFT-based ICPF-FRFT algorithm does not need to perform any parameter search and has high anti-noise performance. These features enable the implementation of the ISAL imaging algorithm in real time.

As a result, the ICPF transform of the optical heterodyne output signal results in spikes only at its chirp-rate slope. The chirp-rate at the peak is $\mu_{k}$ :

$$
\mu_{k}=\underset{\mu}{\operatorname{argmax}}|\operatorname{ICPF}(\mu)|
$$

Calculate the rotation angle $\alpha_{k}$ and FRFT order $p_{k}$ corresponding to the tuning frequency, which are the best FRFT rotation angle and order, respectively. When using discrete FRFT calculations, the signal parameters need to be dimension normalized [29]. The relationship between the chirp-rate and the rotation angle is provided in Equation (28) and the FRFT order is provided in Equation (29):

$$
\mu_{k}=-\cot \left(\alpha_{k}\right) \times \frac{f_{s}^{2}}{N}
$$




$$
p_{k}=\frac{2 \alpha_{k}}{\pi}=\frac{2}{\pi} \operatorname{arccot}\left(-\frac{\mu_{k} f_{s}^{2}}{N}\right)
$$

The optical heterodyne signal in Equation (12) is subjected to $p_{k}$ order FRFT:

$$
\begin{aligned}
& S_{o}\left(u, t_{m}\right)=F^{p_{k}}\left[s_{0}\left(t_{k}, t_{m}\right)\right] \\
& =\left.\sigma_{p} A(u) \cdot \exp \left(j 2 \pi P_{0}\right) \int_{-\infty}^{\infty} \operatorname{rect}\left(\frac{t_{k}-\tau}{T_{p}}\right) \cdot \exp \left[j 2 \pi\left(P_{1}-u \csc \alpha\right) t_{k}\right] \cdot \exp \left[j \pi\left(2 P_{2}+\cot \alpha\right) t_{k}{ }^{2}\right] d t_{k}\right|_{\alpha=\alpha_{k}} \\
& =\sigma_{p} T_{p} A(u) \exp \left(j 2 \pi P_{0}\right) \cdot \operatorname{sinc}\left[T_{p}\left(\frac{u}{\sin \alpha_{k}}-P_{1}\right)\right]
\end{aligned}
$$

where $A(u)=\sqrt{1+j 2 P_{2}} \exp \left(-j 2 \pi P_{2} u^{2}\right)$. From the result, the peak value of the signal is obtained at $u=P_{1} \sin \alpha_{k}$. That is, the range compression of the echo signal is achieved by one FRFT and the phase $\exp \left(j 2 \pi P_{0}\right)$ of the azimuth compression is retained.

\section{Azimuth Imaging Based on ICPF-FRFT}

\subsection{Feature of Azimuth Echo Signal}

In order to facilitate analysis, we assumed that the radar echo has completed range compression and motion compensation, so the echo signal can be converted into a turntable model with centroid as the reference point and the azimuth echo signal at the point can be expressed as:

$$
\mathrm{s}_{p}\left(t_{m}\right)=\sigma \exp \left[-j \frac{4 \pi}{\lambda}\left(x_{p} \sin \theta\left(t_{m}\right)+y_{p} \cos \theta\left(t_{m}\right)\right)\right]
$$

where $\sigma$ is the amplitude of the signal after the motion compensation.

When the target is maneuvering, $\theta(t)$ can be expanded into a function of time $t$ according to Taylor [30] due to the inertia of space targets. For a space target with certain inertia, the ISAL imaging time is shorter and the cumulative rotation angle required by the imaging is smaller, so the motion of the target and radar can approximate to the second-order component, meaning it approximates the uniform acceleration motion.

$$
\theta(t)=\theta_{0}+\omega t+\frac{1}{2 !} \Omega t^{2}
$$

where $\theta_{0}$ is the initial rotation angle, $\omega$ is the rotation angular velocity and $\Omega$ is the rotation angular acceleration.

As the ISAL wavelength is in the order of $\mu \mathrm{m}$, to achieve the imaging resolution of mm magnitudes, the required rotation accumulation angle is in the order of mrad, so the following small angle approximation conditions are satisfied:

$$
\left\{\begin{array}{l}
\sin \theta(t)=\sin \left(\theta_{0}+\omega t+\frac{1}{2} \Omega t^{2}\right) \approx \theta_{0}+\omega t+\frac{1}{2} \Omega t^{2} \\
\cos \theta(t)=\cos \left(\theta_{0}+\omega t+\frac{1}{2} \Omega t^{2}\right) \approx 1-\left(\theta_{0}+\omega t+\frac{1}{2} \Omega t^{2}\right)^{2}
\end{array}\right.
$$

According to Equations (29) and (31), the $P$ point azimuth echo can be approximated to a linear frequency modulated signal.

$$
\mathrm{s}_{p}(t)=\sigma \exp \left[-j \frac{4 \pi}{\lambda}\left(\phi_{0}+f_{a} t+\frac{1}{2} k_{a} t^{2}\right)\right]
$$

where

$$
\begin{gathered}
\phi_{0}=x_{p} \theta_{0}+y_{p}\left(1-\frac{1}{2} \theta_{0}^{2}\right) \\
f_{a}=x_{p} \omega-y_{p} \theta_{0} \omega \\
k_{a}=\frac{1}{2}\left(x_{p} \Omega-y_{p} \omega^{2}-y_{p} \theta_{0} \Omega\right)
\end{gathered}
$$


In practice, multiple scattering points with different intensities are distributed in the same range cell, so the azimuth echo becomes a multicomponent LFM signal with a different LFM rate:

$$
\hat{s}\left(t_{m}\right)=\sum_{i=1}^{I} \sigma_{i} \exp \left(-j \frac{4 \pi}{\lambda}\left(\phi_{0 i}+f_{a i} t_{m}+\frac{1}{2} k_{a i} t_{m}^{2}\right)\right)
$$

where $K$ is the number of scattered points and $\phi_{0 i}, k_{a i}$ and $f_{a i}$ satisfy Equations (35)-(37), respectively.

\subsection{Azimuth Compression Based on ICPF-FRFT}

ICPF can effectively suppress the cross and pseudo peaks caused by the interference of multicomponent signals, so the ICPF-FRFT can be used for imaging azimuth signals but the strong signal components affect the detection of the weak signal components. Therefore, we combined the CLEAN technique with ICPF-FRFT to estimate the strong to weak signals. The frequency modulation slope of the signal was calculated and then FRFT was used to image the signal components of different frequency modulation slopes. The imaging procedure for the azimuth compression based on ICPF-FRFT is shown in Figure 4.

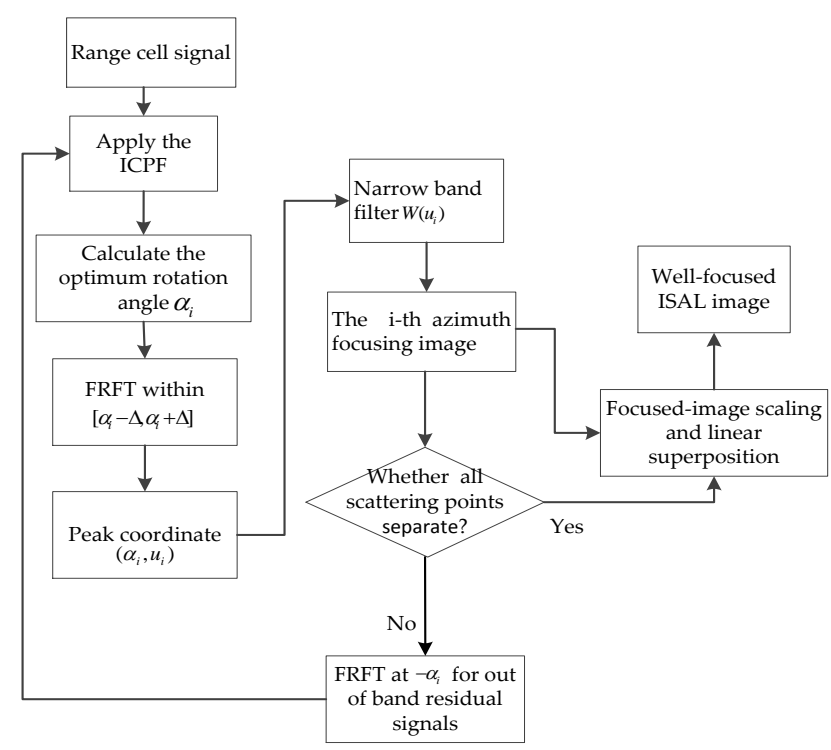

Figure 4. The imaging procedure for the azimuth compression.

The concrete steps are as follows:

Step 1: Calculate the ICPF of a range cell of the echo signal and estimate the frequency modulation rate of the strongest signal component:

$$
\begin{gathered}
\hat{s}\left(t_{m}\right)=\hat{s}\left(\phi_{0 i}, f_{a i}, k_{a i}, t_{m}\right)+\sum_{l=1, l \neq i}^{I} \hat{s}\left(\phi_{0 l}, f_{a l}, k_{a l}, t_{m}\right) \\
k_{a i}=\underset{k_{a}}{\operatorname{argmax}}\left|\operatorname{ICPF}\left[\hat{s}\left(t_{m}\right)\right]\right|=\operatorname{ICPF}\left[\hat{s}\left(\phi_{0 i}, f_{a i}, k_{a i}, t_{m}\right)\right]
\end{gathered}
$$

where $\hat{s}\left(\phi_{0 i}, f_{a i}, k_{a i}, t_{m}\right)$ is the $i$ th signal component.

Step 2: Calculate the best rotation angle $\alpha_{i}$ and the corresponding order $p_{k i}$, according to Equations (28) and (29), respectively. Then, calculate FRFT of the range cell signal in the range 
$\left[\alpha_{i}-\Delta, \alpha_{i}+\Delta\right]$ where $\Delta$ is the calculation error of $\alpha_{i}$. Search the peak to obtain the corresponding position $u_{i}$ :

$$
\begin{aligned}
& \hat{S}_{\alpha}(u)=F^{p_{k i}}\left[\hat{s}\left(\phi_{0 i}, f_{a i}, k_{a i}, t_{m}\right)\right]+\sum_{l=1, l \neq i}^{I} F^{p_{k i}}\left[\hat{s}\left(\phi_{0 l}, f_{a l}, k_{a l}, t_{m}\right)\right]=\hat{S}_{\alpha i}^{i}(u)+\sum_{l=1, l \neq i}^{I} \hat{S}_{\alpha, l}(u) \\
& \left\{u_{i}\right\}=\underset{u}{\operatorname{argmax}}\left[\left|\hat{S}_{\alpha_{i}}(u)\right|\right]
\end{aligned}
$$

Step 3: Separate the peak point by using the CLEAN technique to construct a narrowband filter $W\left(u_{i}\right)$ centered on $u_{i}$. Filter the strongest component and the peak value $\hat{S}_{\alpha i}^{i}(u)$ is considered the azimuth focusing image of the $i$ th component.

$$
\begin{gathered}
\hat{S}_{\alpha_{i}}^{i}(u)=\hat{S}_{\alpha_{i}}(u) W\left(u_{i}\right) \\
\hat{S}_{\alpha, i}(u)=A(u) \sigma_{i} \exp \left(j 2 \pi \phi_{0 i}\right) \sin c\left[T_{p}\left(\frac{u}{\sin \alpha}-f_{a i}\right)\right]
\end{gathered}
$$

where $A(u)=\sqrt{1-j \cot \alpha} \exp \left(j \pi u^{2} \cot \alpha\right)$.

Step 4: Transform the rest of the signal to the time domain using FRFT with a rotation angle of $-\alpha_{i}$.

$$
\hat{s}_{i+1}\left(t_{m}\right)=\int_{-\infty}^{+\infty} \hat{S}_{\alpha_{i}}(u)\left(1-W\left(u_{i}\right)\right) K_{-\alpha_{i}}\left(t_{m}, u\right) d u
$$

Step 5: Repeat the above steps until all the scattered points in the current range cell are separated. This separation can be judged by when the residual signal component energy $E$ of the $i$ th range cell is less than a certain energy threshold $E_{H}$, which is usually $5 \%$ of the original signal [31,32].

Step 6: The target image is obtained by scaling the scattered images $u^{\prime}=u / \sin \alpha$ and stacking them linearly.

Step 7: The 2D ISAL images can be obtained by using the above methods according to the sequence numbers of the range cells.

\section{Imaging Procedure}

For a maneuvering target with approximately uniformly accelerated motion, the ISAL imaging algorithm flow is shown in Figure 5.

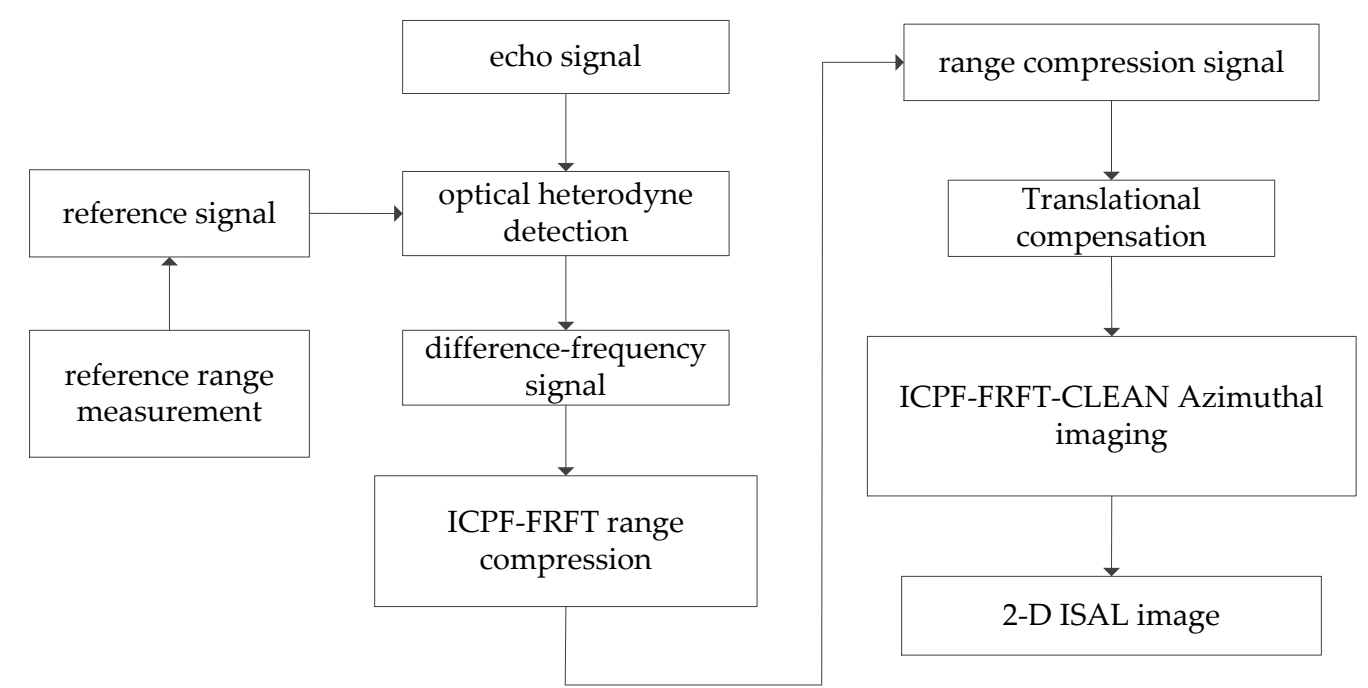

Figure 5. ISAL imaging algorithm flow for maneuvering target. 
The detailed procedure is as follows: (1) Input the received echo signal; (2) Construct the reference signal based on the reference range; (3) Perform the optical heterodyne detection of the echo signal and the reference signal, with the output being the differential frequency signal; (4) Complete range compression with the ICPF-FRFT outlined in Section 3; (5) Translational compensation for the signal after range compression; (6) Azimuthal compression with the ICPF-FRFT-CLEAN outlined in Section 4.2; (7) Output the 2D ISAL image.

\section{Experimental Results of Simulation and Real Data}

In order to verify the effectiveness of the proposed algorithm, simulation and real data experiments were completed. Some other imaging algorithms were considered for comparison.

\subsection{Experimental Simulation Results}

The ladar and target simulation parameters used in the simulation experiment are provided in Table 1, which refers to the scattering point model in Papers [1,11]. The simulation model shown in Figure 6 is a plane model that contains 52 scattering points. The ladar parameters are typical parameters that can be realized and the range resolution was $0.001 \mathrm{~m}$. We assumed that the positional relationship between the ladar and the target was as shown in Figure 1. The target motion parameters were set as the turntable motion parameters as shown in Table 1.

Table 1. Simulation parameters.

\begin{tabular}{cccc}
\hline Radar Parameter & Value & Target Parameter & Value \\
\hline Wavelength $(\mu \mathrm{m})$ & 1.55 & Initial range $(\mathrm{km})$ & 100 \\
bandwidth $(\mathrm{Ghz})$ & 150 & Initial velocity $(\mathrm{m} / \mathrm{s})$ & 100 \\
Pulse width $(\mu \mathrm{s})$ & 100 & Velocity acceleration $\left(\mathrm{m} / \mathrm{s}^{2}\right)$ & 30 \\
PRF () & 3.3 & Angular velocity $(\mathrm{rad} / \mathrm{s})$ & 0.005 \\
Range sampling number & 256 & Angular acceleration $\left(\mathrm{rad} / \mathrm{s}^{2}\right)$ & 0.01 \\
Pulse number & 512 & Angular acceleration rate $\left(\mathrm{rad} / \mathrm{s}^{3}\right)$ & 0.006 \\
Processing time $(\mathrm{s})$ & 0.155 & - & - \\
\hline
\end{tabular}

Figure 7 is the smooth pseudo Wigner distribution (SPWVD) time frequency graph of the 128th pulse echo. The ISAL single echo signal is a multicomponent LFM signal with the same modulation frequency, which confirms the analysis of the echo signal in Section 2. Therefore, the compression of all scattering points can be accomplished through one compression of a range. Figure 8 shows the 128th pulse range compression result using the DFT method. From the display results, the direct use of DFT compression results in a serious dispersion effect of the range image and the scattering points of the adjacent resolution units form. Serious mutual interference occurs for scattering points of adjacent resolution units. Figure 9 shows the 128th pulse range compression result using the ICPF-FRFT method proposed in this paper. As seen in the figure, the results show that a better compression effect was achieved and the range dispersion was eliminated. 


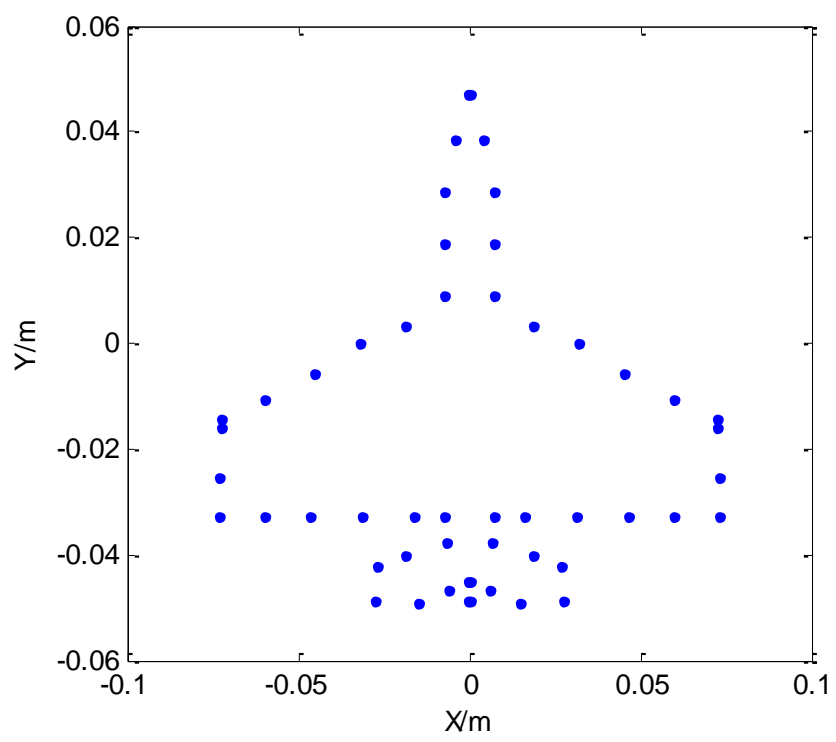

Figure 6. Simulation model of aircraft.

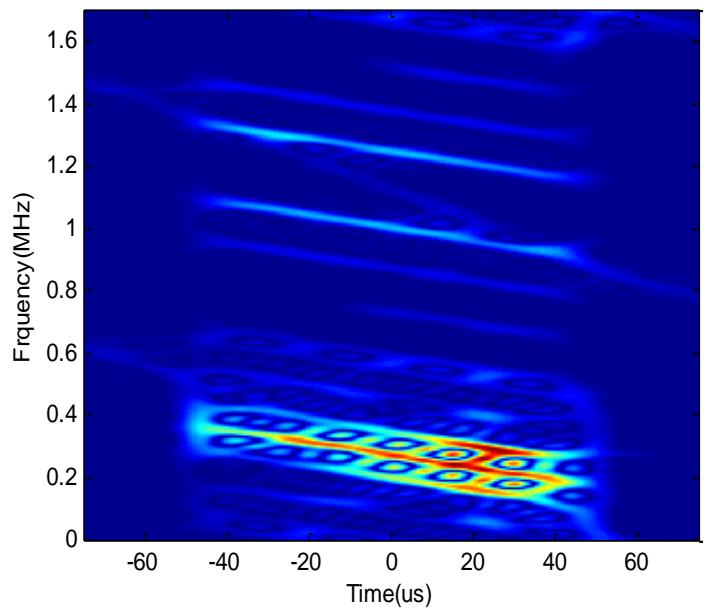

Figure 7. The 128th pulse's smooth pseudo Wigner distribution (SPWVD).

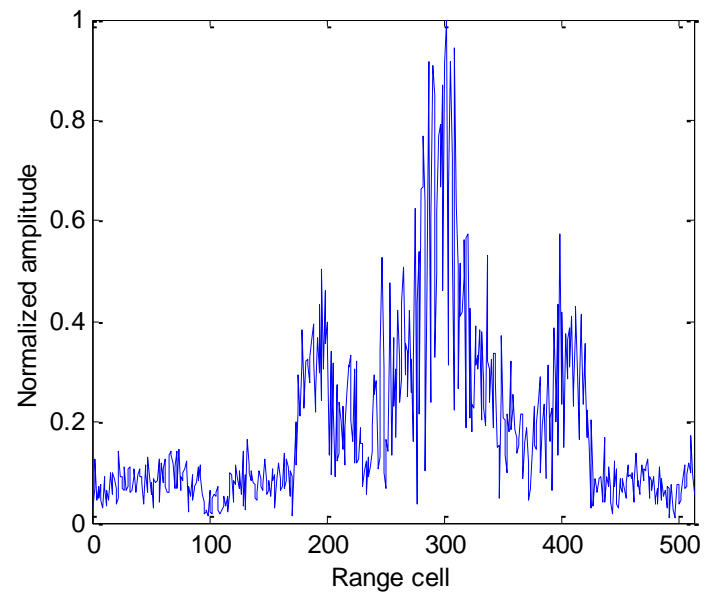

Figure 8. The 128th pulse's range compression via Discrete Fourier transform (DFT). 


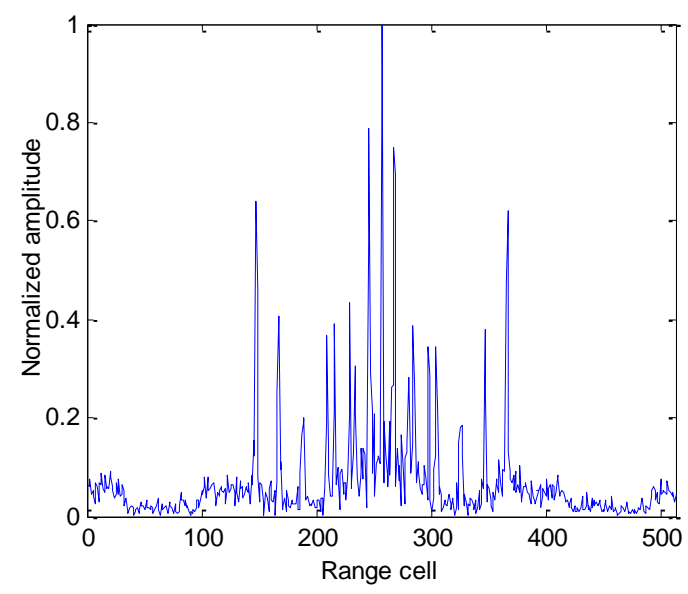

Figure 9. The 128th pulse's range compression via integral cubic phase function-fractional Fourier transform (ICPF-FRFT).

Figure 10 provides the SPWVD time frequency graph of the 79th (the lower edge of the aircraft) and 128th (the aircraft range center) range cell. From the diagram, due to the short ISAL imaging time, the target azimuth echo signals can be approximated as a multi-component LFM signal, even if there is a third order rotational component (angle acceleration rate) in the target. This demonstrates a slope with different slopes on the time frequency graph, which is the same as the previous theoretical analysis. So, dividing the scattering imaging into scattering points on different range cells was necessary.

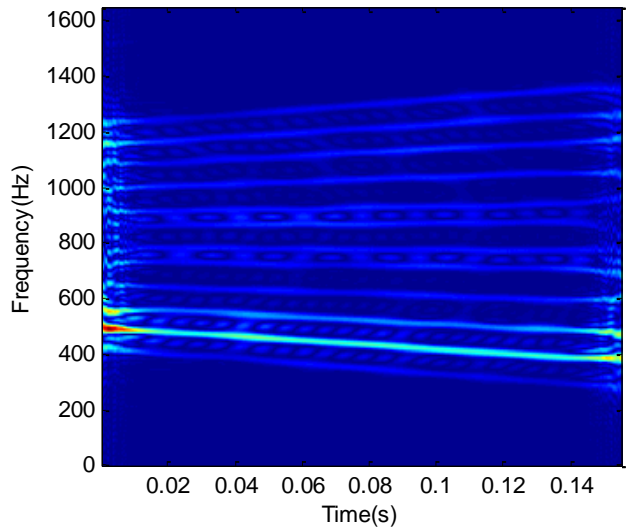

(a)

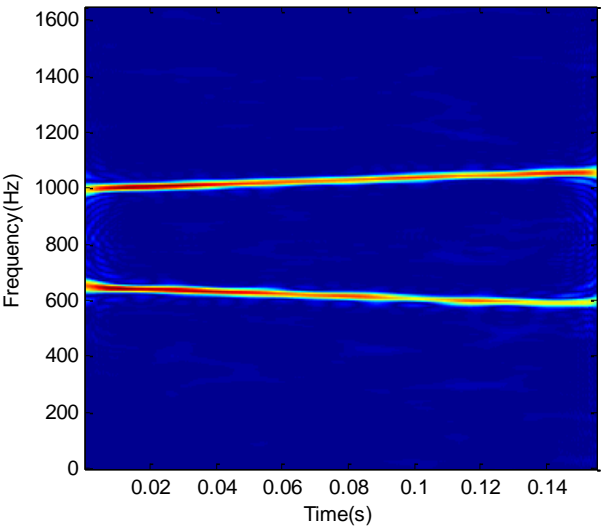

(b)

Figure 10. Range cell's SPWVD: (a) the 79th range cell and (b) the 128th range cell.

Figure 11a shows the result of traditional DFT azimuthal compression, which highlights that the scattered points from the center of the azimuth were seriously defocused-a poor imaging result. For comparison, we also provide three instantaneous Doppler (RID) imaging results based on STFT, WVD and SPWVD, as shown in Figure $11 \mathrm{~b}-\mathrm{d}$, respectively. The image results used the 24th frame, which was $t=0.116 \mathrm{~s}$. From the results of STFT in Figure $11 \mathrm{~b}$, the time-frequency resolution is affected by the window function and the azimuth defocus was severe. From Figure 11c, WVD can improve upon the time-frequency resolution but since the azimuth echo is a multi-component signal, the imaging result produces a cross term, so the imaging results are poorly readable and cannot identify the target. The SPWVD provides windowing and smoothing of WVD, so it weakens the cross terms but the time-frequency resolution also decreases. From Figure 11d, we can verify that the SPWVD has no cross-scattering point compared to the result of Figure 11c but the resolution is reduced. From the four results in Figure 11, the direct imaging range-Doppler (RD) algorithm and the 
three range-instantaneous Doppler (RID) imaging methods cannot achieve better imaging results and the azimuth defocus still exists.

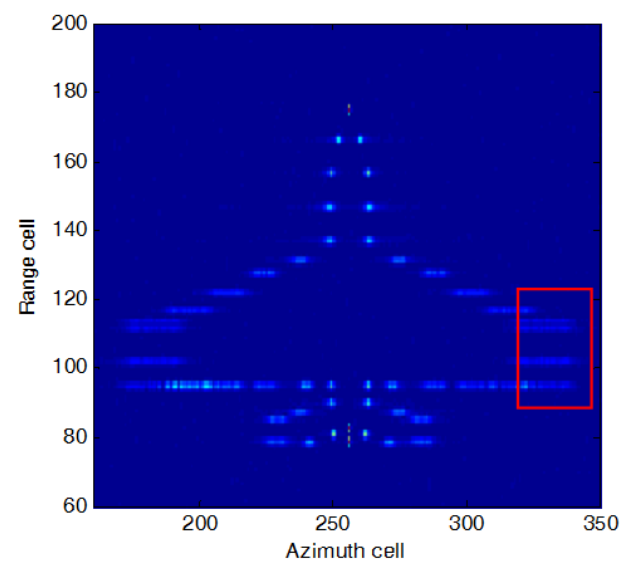

(a)

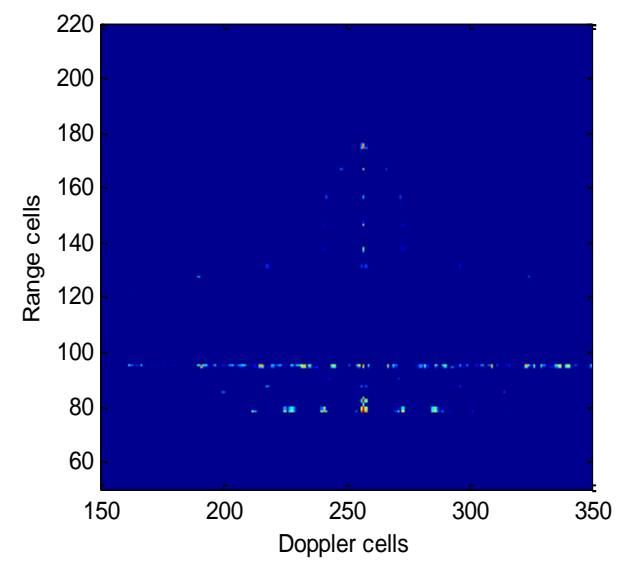

(c)

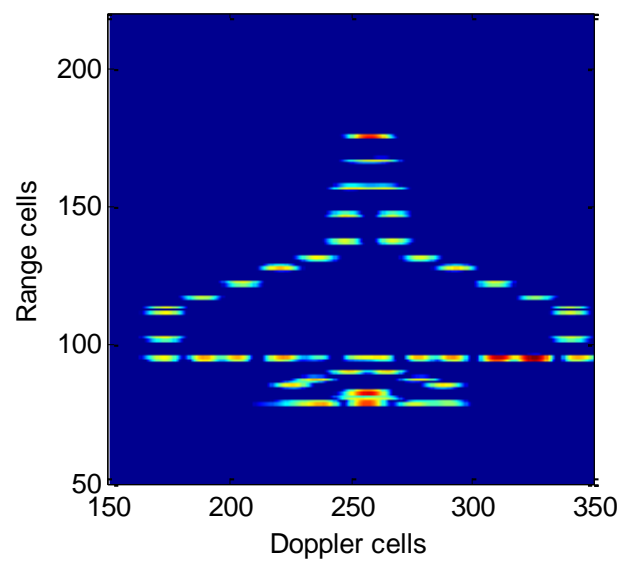

(b)

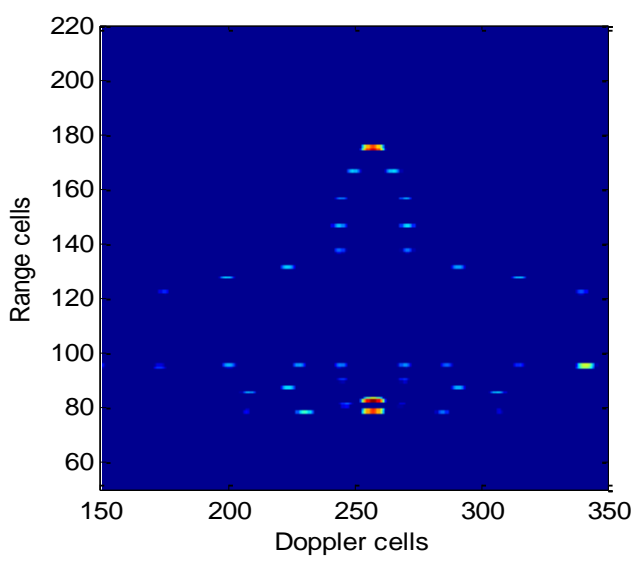

(d)

Figure 11. Azimuth compression via classical methods: (a) direct DFT, (b) RID image based on STFT, (c) RID image based on WVD and (d) RID image based on SPWVD.

Figure 12 is the result of azimuth compression using ICPF-FRFT. In Figure 12a, the CLEAN technique is not used, whereas it is applied in Figure 12b. Figure 12a shows that the imaging results of a single scattering point on a certain range cell are good. With multiple scattering points, the strong signal suppresses the separation of the weak signal due to the different signal intensities. Most of the signal is strong and is the signal of its side lobe component, and the weak signal is missing. Figure $12 \mathrm{~b}$ shows that when the CLEAN technique was used to separate the scattering points on different range cells from strong to weak separation imaging, a better focusing effect was achieved. The diagram demonstrates that this method can effectively separate the scattering points of two range cells from each other, proving the effectiveness of the square method and further illustrates that the ISAL can be applied in real situations. High precision (millimeter level) imaging of space targets is now being performed.

Figure 13a-c are schematic diagrams of the separation of the first three peaks of the 95 range cell on the lower wing of the aircraft. The left image is the search process of the FRFT peak in the ICPF estimation error, the right side is the Clean processing for the peak point, the red frame is a narrow band filter and the frame content is the transverse focus image. Through this process, the azimuth scattering points with different intensities were separated and appropriately imaged. 


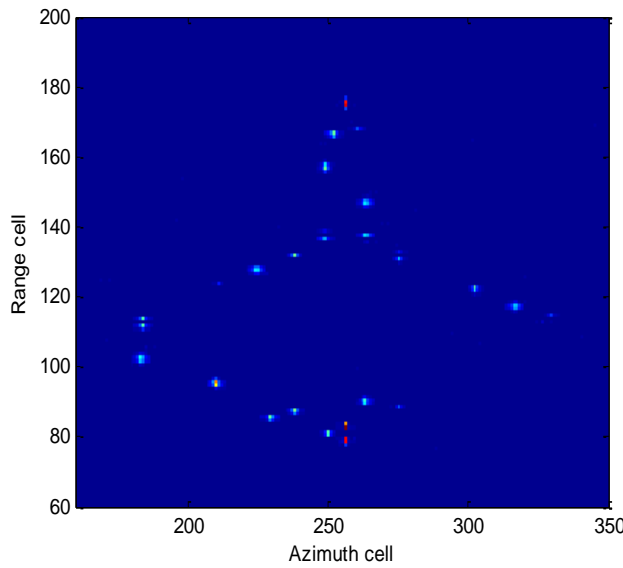

(a)

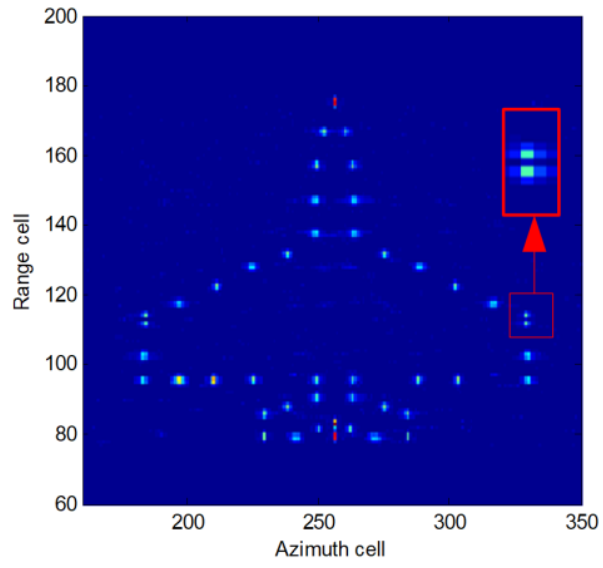

(b)

Figure 12. Azimuth compression via ICPF-FRFT: (a) without the CLEAN technique and (b) with the CLEAN technique.
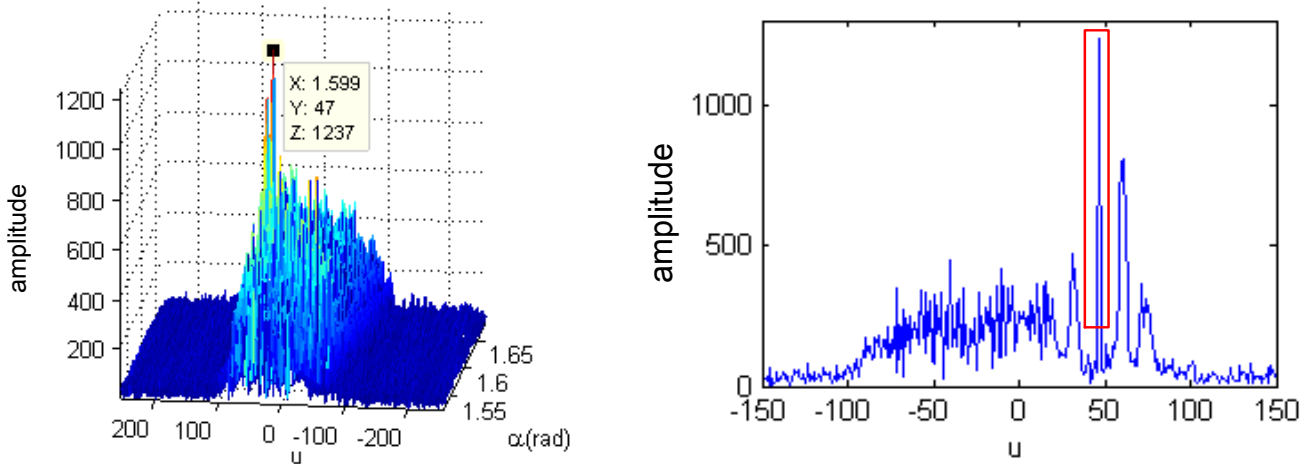

(a)
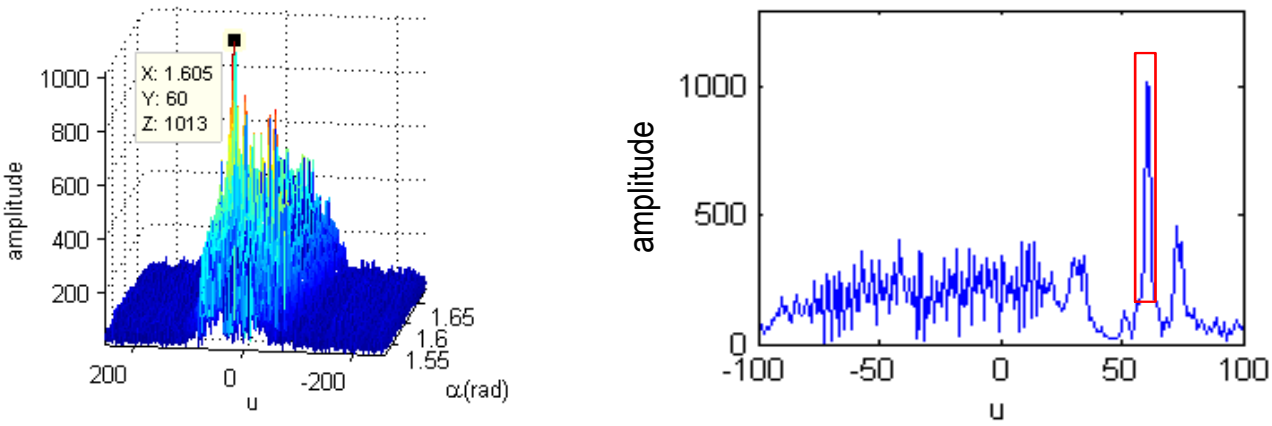

(b)
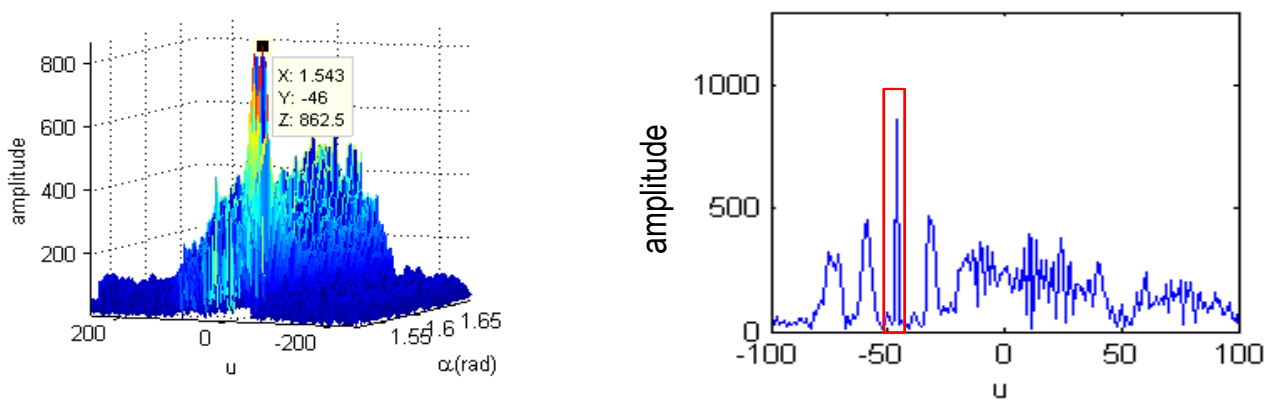

(c)

Figure 13. Separation imaging of the 95th range cell: (a) peak one, (b) peak two and (c) peak three. 
In order to quantitatively evaluate the effectiveness of the proposed ICPF-FRFT algorithm, image entropy, contrast and running time were used to illustrate the imaging quality of the algorithm. Suppose the acquired ISAL image is $f(n, k)$, where $n$ and $k$ are the range and azimuth number of the sampling unit, respectively. The definition of image entropy is:

$$
\begin{gathered}
E=-\sum_{k=1}^{K} \sum_{n=1}^{N} \frac{|f(n, k)|^{2}}{F} \ln \frac{|f(n, k)|^{2}}{F} \\
F=\sum_{k=1}^{K} \sum_{n=1}^{N}|f(n, k)|^{2}
\end{gathered}
$$

where $F$ is the total energy of the ISAL image. The image entropy is small when the image is well-focused. Conversely, a large image entropy indicates that the compensation effect is worse.

The definition of image contrast is:

$$
C=\frac{\sqrt{E\left\{[|f(n, k)|-E(|f(n, k)|)]^{2}\right\}}}{E(|f(n, k)|)}
$$

where $E(\cdot)$ represents the average operation. The image contrast is large when the image is well-focused. Conversely, a small image entropy indicates that the compensation effect is worse.

The results of the proposed ICPF-FRFT algorithm compared with the RD algorithm, FRFT algorithm, the ICPF-FRFT algorithm without CLEAN technique and three RID imaging methods based on STFT, WVD and SPWVD (Table 2). From the table, the algorithm proposed in this paper has smaller image entropy and a larger image contrast than the other algorithms, which shows that the image quality of the algorithm proposed in this paper is better. Notably, although all the indexes of the CLEAN technique are better, the loss of the scattering points cannot correctly reflect the distribution of the target scattering point, so the imaging quality was not the best. When the FRFT imaging algorithm with a small step size is performed directly, the result can reach an image entropy and contrast close to that of the proposed ICPF-FRFT algorithm paper but considerable computation time is required, indicating that the proposed algorithm is more efficient.

Table 2. Comparison of simulation aircraft imaging results.

\begin{tabular}{cccccccc}
\hline Imaging Algorithm & RD & STFT & WVD & SPWVD & FRFT & No-CLEAN & ICPF-FRFT \\
\hline Image entropy & 8.0546 & 9.2598 & 7.6822 & 6.0802 & 5.7540 & 4.1374 & 5.2629 \\
Contrast ratio & 1.6678 & 1.3632 & 3.4347 & 8.4177 & 10.8059 & 29.6294 & 13.0397 \\
Running time (s) & 0.2066 & 3.3042 & 4.9585 & 215.8427 & 77.0294 & 2.3453 & 3.8554 \\
\hline
\end{tabular}

\subsection{Experimental Results of Real Data}

Since no ISAL data have been published to date, the research on ISAL at this stage is mainly based on simulation data to verify algorithms. However, considering the problem of azimuth Doppler time-varying when imaging a maneuvering target in ISAR, ISAR is consistent with ISAL imaging in the pursuit of azimuth focusing. Therefore, the ISAL azimuth imaging algorithm based on ICPF-FRFT is also suitable for imaging ISAR maneuvering targets but the radar signal bandwidth in ISAR is much smaller than ISAL, so the spread over the range can be ignored. To further validate the effectiveness of the algorithm, the publicly available Boeing B727 ISAR aircraft data from Victor C. Chen of the U.S. Naval Research Laboratory (Washington, DC, USA) was used for experimental verification [33]. The data included 256 continuous pulses with a carrier frequency of $9 \mathrm{GHz}$, a bandwidth of $150 \mathrm{MHz}$ and a pulse repetition rate of $20 \mathrm{kHz}$. Range compression and the motion compensation for the data were completed. 
The imaging results using the imaging method proposed in this paper and other comparison methods described in the previous section are shown in Figure 14. The evaluation indexes of each imaging result are shown in Table 3. As can be seen from Figure 14a, the azimuth defocusing that occurred when using the RD algorithm was severe. From Figure $14 \mathrm{~b}-\mathrm{d}$, the RID imaging results are related to the time-frequency method used, in which the time-frequency resolution of STFT was the worst and WVD had the highest time-frequency resolution but the cross-term was the most serious and SPWVD was somewhere in between. The result of the notable time-frequency imaging method shows that as the azimuth Doppler dynamically changes, the results displayed at different azimuths are different. In addition, some weak scattering point energy loss occurs, as shown in the wing part of the figure. Some scattering points are missing. It can be seen from Figure $14 \mathrm{e}-\mathrm{g}$ that all three imaging methods can effectively improve the azimuth focusing effect but the direct FRFT requires a long computation time to achieve the same focusing effect as ICPF-FRFT. However, for the No-CLEAN technique, although all the indicators are superior, this algorithm only focuses on the strong scattering point, resulting in a lack of partial scattering points. Considering the minimum entropy, contrast and running time, the ICPF-FRFT algorithm is optimal, which is consistent with the results of the previous simulation analysis.

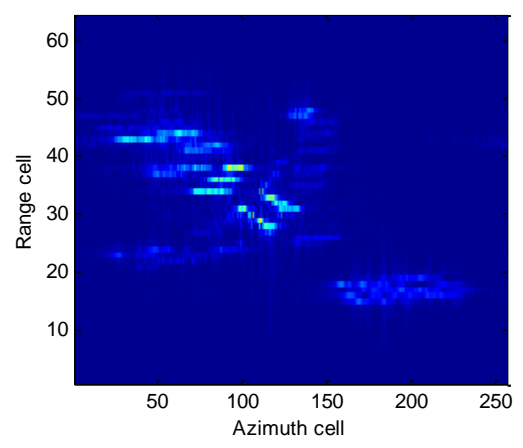

(a)

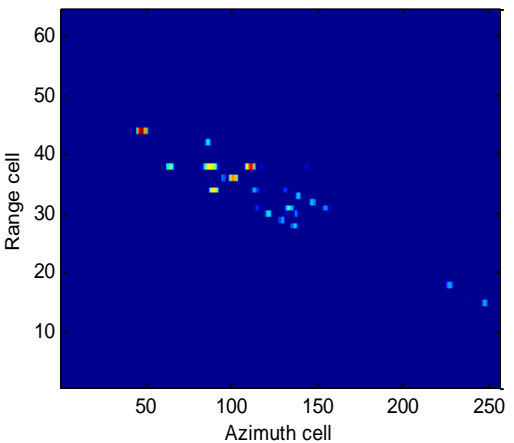

(c)

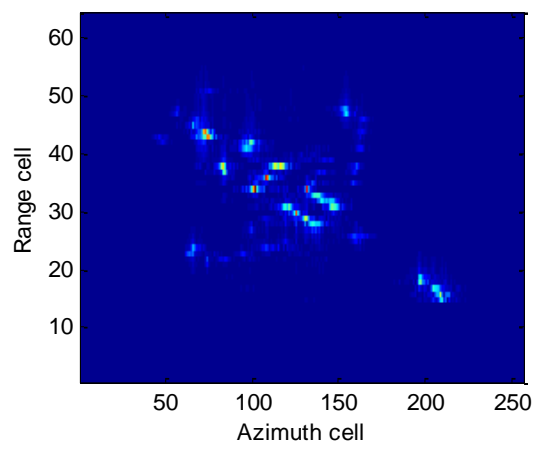

(e)

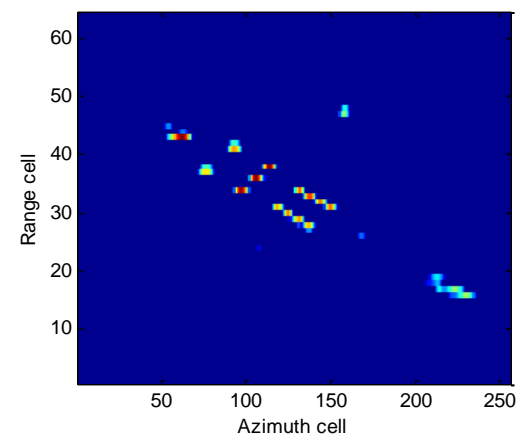

(b)

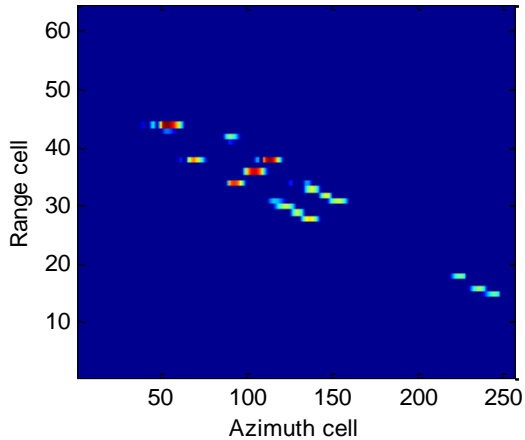

(d)

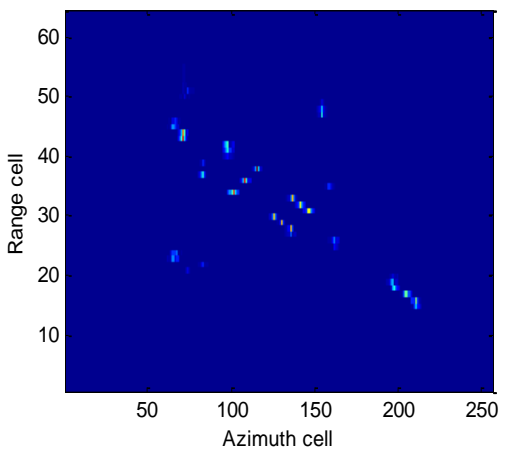

(f)

Figure 14. Cont. 


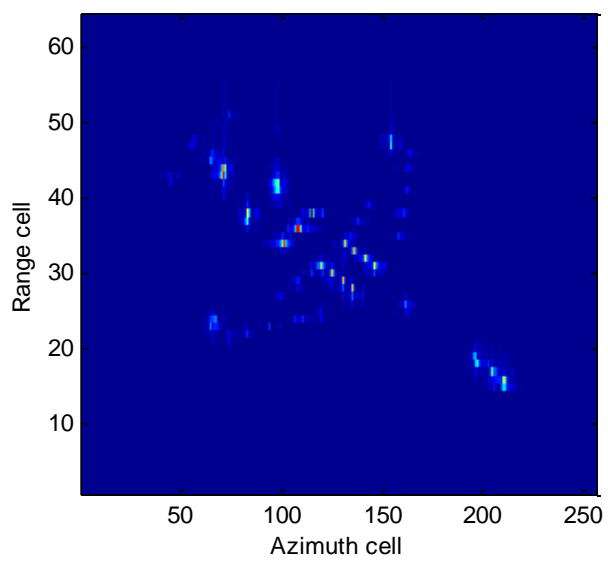

(g)

Figure 14. Inverse synthetic aperture radar (ISAR) imaging results: (a) RD algorithm, (b) STFT algorithm, (c) WVD, (d) SPWVD, (e) FRFT, (f) No-CLEAN technique and (g) ICPF-FRFT algorithm. The simulated echo pulses of Boeing-727 shown in Figure 14 are available online at http:/ /www.mdpi.com/20724292/10/4/593/s1.

Table 3. Comparison results of the Boeing-727 images.

\begin{tabular}{cccccccc}
\hline Imaging Algorithm & RD & STFT & WVD & SPWVD & FRFT & No-CLEAN & ICPF-FRFT \\
\hline Image entropy & 6.6428 & 6.1032 & 5.7609 & 4.8711 & 5.5242 & 3.9852 & 4.2940 \\
Contrast ratio & 2.3968 & 2.8949 & 3.6200 & 6.4915 & 4.8906 & 12.7516 & 7.5289 \\
Running time (s) & 0.0014 & 0.2350 & 0.4022 & 9.6358 & 24.0325 & 1.0412 & 1.2939 \\
\hline
\end{tabular}

\section{Conclusions}

ISAL can meet the high precision and quasi real-time imaging requirements for targets. However, due to the use of ultra-high carrier frequency and large bandwidth signals, the ISAL radar's target echo signal produces distortion and a 1D dispersion profile. In addition, when the target moves, the radar signal echo direction changes to Doppler. To address these issues, an ISAL imaging algorithm based on ICPF-FRFT was proposed for space maneuvering targets, which was able to quickly image uniformly accelerated motion. The algorithm first uses the ICPF algorithm based on NUFFT computing to quickly estimate the frequency modulation rate of the echo signal and then uses FRFT to compress the range image at the best rotation angle and eliminate the range dispersion. After motion compensation, the ICPF-FRFT and CLEAN technique are used again to separate the strong and weak scattering points and solve the azimuth defocusing problem. The validity of the method was verified with a simulation experiment of an aircraft scatter point model and Boeing-727 data.

Author Contributions: Y.L. and L.Q. conceived and designed the method; Y.W. guided the students to complete the research; Y.L. performed the simulation and experiment tests; H.W., J.J. and Y.S. helped in the simulation and experiment tests; and Y.L. wrote the paper.

Funding: The authors are grateful for the financial support received from the State 863 Project of China (No. 2014AA7113016) and the Research Project of the State Key Laboratory of Complex Electromagnetic Environment Effects on Electronics and Information Systems (No. 2017Z0203B).

Conflicts of Interest: The authors declare no conflict of interest.

\section{References}

1. He, J.; Zhang, Q.; Yang, X.Y.; Luo, Y.; Zhu, X.P. High resolution imaging algorithm for inverse synthetic aperture imaging LADAR. Syst. Eng. Electron. 2011, 33, 1750-1755. 
2. Liu, L.R. Synthetic aperture laser imaging radar (I): Defocused and phase biased telescope for reception antenna. Acta Opt. Sin. 2008, 28, 997-1000.

3. Beck, S.M.; Buck, J.R.; Buell, W.F.; Dickinson, R.P.; Kozlowski, D.A.; Marechal, N.J.; Wright, T.J. Synthetic-aperture imaging laser radar: Laboratory demonstration and signal processing. Appl. Opt. 2005, 44, 7621-7629. [CrossRef] [PubMed]

4. Crouch, S.; Barber, Z.W. Laboratory demonstrations of interferometric and spotlight synthetic aperture ladar techniques. Opt. Express 2012, 20, 24237-24246. [CrossRef] [PubMed]

5. Turbide, S.; Marchese, L.; Terroux, M.; Bergeron, A. Synthetic aperture ladar concept for infrastructure monitoring. In Electro-Optical Remote Sensing, Photonic Technologies and Applications VIII; and Military Applications in Hyperspectral Imaging and High Spatial Resolution Sensing II; International Society for Optics and Photonics: Bellingham, WA, USA, 2014.

6. Barber, Z.W.; Dahl, J.R. Synthetic aperture ladar imaging demonstrations and information at very low return levels. Appl. Opt. 2014, 53, 5531-5537. [CrossRef] [PubMed]

7. Trahan, R.; Nemati, B.; Zhou, H.; Shao, M.; Hann, I.; Schulze, W. Low-CNR inverse synthetic aperture LADAR imaging demonstration with atmospheric turbulence. In Long-Range Imaging; International Society for Optics and Photonics: Bellingham, WA, USA, 2016.

8. Luan, Z.; Sun, J.; Zhou, Y.; Wang, L.; Yang, M.; Liu, L. Down-looking synthetic aperture imaging ladar demonstrator and its experiments over $1.2 \mathrm{~km}$ outdoor. Chin. Opt. Lett. 2014, 12, 111101. [CrossRef]

9. Ikram, M.Z.; Abed-Meraim, K.; Hua, Y. Estimating the parameters of chirp signals: An iterative approach. IEEE Trans. Signal Process. 2002, 46, 3436-3441. [CrossRef]

10. Barbarossa, S.; Scaglione, A.; Giannakis, G.B. Product high-order ambiguity function for multicomponent polynomial-phase signal modeling. IEEE Trans. Signal Process. 1998, 46, 691-708. [CrossRef]

11. Xia, X.G. Discrete chirp-Fourier transform and its application to chirp rate estimation. IEEE Trans. Signal Process. 2000, 48, 3122-3133.

12. Djurović, I.; Simeunović, M.; Wang, P. Cubic phase function: A simple solution to polynomial phase signal analysis. Signal Process. 2017, 135, 48-66. [CrossRef]

13. Chen, V.C.; Miceli, W.J. Time-varying spectral analysis for radar imaging of maneuvering targets. IET Proc. Radar Sonar Navig. 1998, 145, 262-268. [CrossRef]

14. Xing, M.D.; Wu, R.B.; Li, Y.C.; Bao, Z. New ISAR imaging algorithm based on modified Wigner Ville distribution. IET Proc. Radar Sonar Navig. 2009, 3, 70-80. [CrossRef]

15. Trintinalia, L.; Ling, H. Joint time-frequency ISAR using adaptive processing. IEEE Trans. Antennas Propag. 1997, 45, 221-227. [CrossRef]

16. Lao, G.; Yin, C.; Ye, W.; Sun, Y.; Li, G. A frequency domain extraction based adaptive joint time frequency decomposition method of the maneuvering target radar echo. Remote Sens. 2018, 10, 266. [CrossRef]

17. Tao, R.; Deng, B.; Wang, Y. Research progress of the fractional Fourier transform in signal processing. Sci. China Inf. Sci. 2006, 49, 1-25. [CrossRef]

18. Wang, B.; Xu, S.; Wu, W.; Hu, P.; Chen, Z. Adaptive ISAR imaging of maneuvering targets based on a modified Fourier transform. Sensors 2018, 18, 1370. [CrossRef] [PubMed]

19. Attia, E.H. Data-adaptive motion compensation for synthetic aperture LADAR. In Proceedings of the 2004 IEEE Aerospace Conference, Big Sky, MT, USA, 6-13 March 2004.

20. Shapiro, J.H.; Capron, B.A.; Harney, R.C. Imaging and target detection with a heterodyne-reception optical radar. Appl. Opt. 1981, 20, 3292-3313. [CrossRef] [PubMed]

21. Song, J.; Liu, Y.; Zhu, X. Parameters estimation of LFM signals by interpolation based on FRFT. Syst. Eng. Electron. 2011, 33, 2188-2193.

22. O'Shea, P. A new technique for instantaneous frequency rate estimation. IEEE Signal Process. Lett. 2002, 9, 251-252. [CrossRef]

23. Wang, P.; Li, H.; Djurović, I.; Himed, B. Integrated cubic phase function for linear FM signal analysis. IEEE Trans. Aerosp. Electron. Syst. 2010, 46, 963-977. [CrossRef]

24. Su, J.; Tao, H.; Xie, J.; Sun, Y.; Li, G. Imaging and Doppler parameter estimation for maneuvering target using axis mapping based coherently integrated cubic phase function. Digital Signal Process. 2018, 10, 266. [CrossRef]

25. Li, H.; Qin, Y.L.; Li, Y.P.; Wang, H.Q.; Li, X. Analysis of multi-component LFM signals by the integrated quadratic phase function. J. Electron. Inf. Technol. 2012, 28, 926-931. 
26. Liu, Q.H.; Nguyen, N. An accurate algorithm for nonuniform fast Fourier transforms (NUFFT's). IEEE Microw. Guided Wave Lett. 2002, 8, 18-20. [CrossRef]

27. O'Shea, P. Improving polynomial phase parameter estimation by using nonuniformly spaced signal sample methods. IEEE Trans. Signal Process. 2012, 7, 3405-3414. [CrossRef]

28. Xing, M.; Wu, R.; Lan, J.; Bao, Z. Migration through resolution cell compensation in ISAR imaging. IEEE Geosci. Remote Sens. Lett. 2004, 1, 141-144. [CrossRef]

29. Simeunović, M.; Djurović, I. Non-uniform sampled cubic phase function. Signal Process. 2014, 101, 99-103. [CrossRef]

30. Wang, C.; Wang, Y.; Li, S.B. Inverse synthetic aperture radar imaging of ship targets with complex motion based on match Fourier transform for cubic chirps model. IET Radar Sonar Navig. 2013, 7, 994-1003. [CrossRef]

31. Wang, Y.; Lin, Y. ISAR imaging of non-uniformly rotating target via range-instantaneous Doppler derivatives algorithm. IEEE J. Sel. Top. Appl. Earth Obs. Remote Sens. 2014, 7, 167-176. [CrossRef]

32. Zheng, J.; Liu, H.; Liu, Z.; Liu, Q.H. ISAR imaging of ship targets based on an integrated cubic phase bilinear autocorrelation function. Sensors 2017, 17, 498. [CrossRef] [PubMed]

33. B727S.mat. Available online: http:/ / www.mdpi.com/2072-4292/10/4/593/s1 (accessed on 12 April 2018).

(C) 2018 by the authors. Licensee MDPI, Basel, Switzerland. This article is an open access article distributed under the terms and conditions of the Creative Commons Attribution (CC BY) license (http:/ / creativecommons.org/licenses/by/4.0/). 